\section{RMD Open}

Rheumatic \& Musculoskeletal Diseases

\title{
Re-treatment with abatacept plus methotrexate for disease flare after complete treatment withdrawal in patients with early rheumatoid arthritis: 2-year results from the AVERT study
}

To cite: Emery P, Burmester GR, Bykerk VP, et al. Retreatment with abatacept plus methotrexate for disease flare after complete treatment withdrawal in patients with early rheumatoid arthritis: 2-year results from the AVERT study. RMD Open 2019;5:e000840. doi:10.1136/ rmdopen-2018-000840

- Prepublication history and additional material for this paper are available in online. To view these files, please visit the journal online (http://dx.doi. org/10.1136/rmdopen-2018000840).

Received 11 October 2018 Revised 6 December 2018 Accepted 8 January 2019

Check for updates

(C) Author(s) (or their employer(s)) 2019. Re-use permitted under CC BY-NC. No commercial re-use. See rights and permissions. Published by BMJ.

For numbered affiliations see end of article.

Correspondence to Dr Paul Emery; p.emery@leeds.ac.uk

\section{ABSTRACT}

Objectives To complete reporting of outcomes after total withdrawal of all rheumatoid arthritis (RA) therapy and retreatment after flare in Assessing Very Early Rheumatoid arthritis Treatment study (NCT01142726).

Methods Patients with early RA were initially randomised to double-blind, weekly subcutaneous abatacept plus methotrexate, or abatacept or methotrexate monotherapy. At month 12, patients with Disease Activity Score (DAS)28 $C$ reactive protein $(\mathrm{CRP})<3.2$ had all $\mathrm{RA}$ treatments rapidly withdrawn and were observed for $\leq 12$ months or until flare. After $\geq 3$ months' withdrawal, patients with protocoldefined RA flare received open-label abatacept plus methotrexate for 6 months (re-treatment).

Results Proportion of patients in DAS28-CRP-defined remission remained numerically higher in original abatacept plus methotrexate and abatacept arms versus methotrexate arm up to day 253 of withdrawal. At the end of the withdrawal period, few patients remained in remission across all arms: 9/73 (12.3\%), 7/50 (14.0\%) and $6 / 53(11.3 \%)$, respectively. For patients entering retreatment, after 6 months' re-treatment, 95/124 (76.6\%) and 78/124 (62.9\%) patients achieved DAS28-CRP $<3.2$ and $<2.6$, respectively; mean changes in DAS28-CRP and Health Assessment Questionnaire-Disability Index scores from re-treatment baseline were -2.87 and 0.76 , respectively. Re-treatment was well tolerated; exposureadjusted infection rates per 100 patient-years were lower with abatacept plus methotrexate during withdrawal (7.2) and re-treatment (17.2) versus initial treatment periods of months 0-6 (116.6) and 6-12 (64.6).

Conclusions Most patients flared within 6 months of therapy withdrawal and few sustained major responses for 1 year. Re-treatment with abatacept plus methotrexate was effective and well tolerated in this controlled setting.

\section{INTRODUCTION}

In early rheumatoid arthritis (RA), a window may exist during which intensive treatment can alter the disease course, leading to improved long-term outcomes. ${ }^{12}$ For patients

\section{Key messages}

What is already known about this subject?

- Abatacept in combination with methotrexate induces remission more often than methotrexate alone after 12 months of treatment, with a greater proportion of patients with rheumatoid arthritis (RA) maintaining remission over 6 months following treatment withdrawal.

What does this study add?

- These final results of the Assessing Very Early Rheumatoid arthritis Treatment (AVERT) study demonstrate that, after receiving only 12 months of disease-modifying antirheumatic drug therapy before withdrawal of all RA treatments, the majority of patients will experience a flare within 6 months of treatment withdrawal and very few will sustain major responses for 1 year.

- The study provides the most complete data on re-treatment with abatacept plus methotrexate showing that this combination can restore remission or low disease activity in many patients who experienced a flare after treatment withdrawal, with good tolerability.

How might this impact on clinical practice?

- This is the first study to examine the effects of complete and rapid removal of all RA therapy, creating data that will inform future efforts to develop offdrug remission strategies.

achieving remission within this window, tapering or withdrawal of treatment may offer certain advantages if remission is sustained..$^{3-5}$ In order to develop biologic-free or even drug-free remission strategies, ${ }^{3}$ we must better understand the ability of current therapies to sustain long-term off-drug remission, including the management of disease flares. 


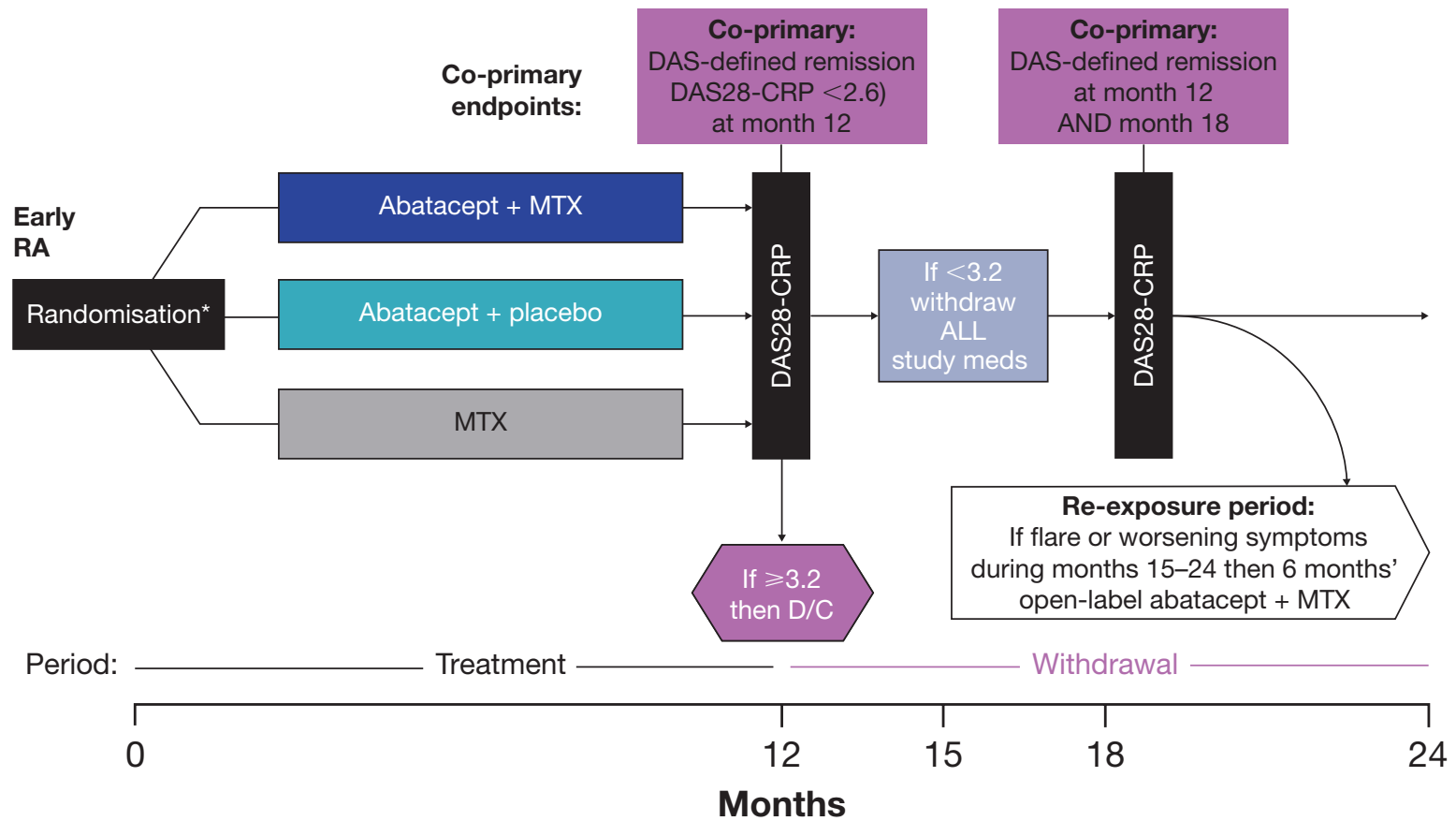

Figure 1 Study design. *Randomisation stratified by corticosteroid use at baseline. DAS28-CRP, Disease Activity Score 28-C reactive protein; D/C, discontinuation; MTX, methotrexate; RA, rheumatoid arthritis. Reprinted from Emery $\mathrm{P}$, et al. Ann Rheum Dis 2015;74:19-26, which is published under Creative Commons license CC BY-NC 4.0 (https://creativecommons.org/licenses/ by-nc/4.0/legalcode).

In the Assessing Very Early Rheumatoid arthritis Treatment (AVERT) study, treatment-naïve patients with early RA were randomised to either subcutaneous abatacept plus methotrexate, subcutaneous abatacept or methotrexate monotherapy for 1 year followed by complete withdrawal of all RA-specific therapeutic agents, including corticosteroids, and then monitored for flares before re-treating with abatacept plus methotrexate. As previously reported, it demonstrated the benefits, including MRI improvements, of abatacept plus methotrexate versus methotrexate alone in inducing remission at 12 months and maintaining remission over the first 6 months after withdrawal of all RA treatment. ${ }^{67}$ This report completes the description of the efficacy and safety outcomes over the full 12-month treatment withdrawal period and the benefits of 6 months of re-treatment after an RA flare. It also explores which clinical characteristics were associated with flare or with regaining disease control after re-treatment.

\section{METHODS}

\section{Study design and patient population}

Methodology of the phase IIIb AVERT study (NCT01142726) was published previously (figure 1). ${ }^{6}$ In the initial 12-month, double-blind treatment period, patients were randomised (1:1:1) to subcutaneous abatacept plus oral methotrexate, abatacept monotherapy plus oral placebo, or methotrexate monotherapy plus subcutaneous placebo. After 12 months, patients with Disease Activity Score (DAS) 28 C reactive protein (CRP) $<3.2$ could enter a 12-month withdrawal period and all RA treatments were stopped-abatacept immediately, and methotrexate and corticosteroids tapered over 1 month. Patients with protocol-defined RA flare after month 15 could receive open-label subcutaneous abatacept plus methotrexate in a 6-month re-treatment period. RA flare was defined as $\geq 2$ of the following after the first 3 months of the withdrawal period: doubling of tender and swollen joint counts, increase in DAS28-CRP $\geq 1.2$ (both from month 12) or investigator's judgement of RA flare. Abatacept was administered at $125 \mathrm{mg}$ /week and methotrexate titrated to $10-20 \mathrm{mg}$ /week.

Eligibility criteria included age $\geq 18$ years, active clinical synovitis of $\geq 2$ joints for $\geq 8$ weeks, persistent RA symptoms for $\leq 2$ years, DAS28-CRP $\geq 3.2$, anticyclic citrullinated peptide 2 (anti-CCP2) antibody positivity and methotrexate naïve/minimum exposure. Patients receiving oral corticosteroids had to be on a stable dose $(\leq 10 \mathrm{mg}$ / day) at baseline and maintain that dose until month 12; randomisation was stratified by baseline corticosteroid use (yes/no).

\section{Assessments and statistical analyses}

Proportions of patients in the withdrawal period with remission, defined as DAS28-CRP $<2.6$, Clinical Disease Activity Index (CDAI) $\leq 2.8$, Simplified Disease Activity Index (SDAI) $\leq 3.3$ and Boolean criteria, ${ }^{8}$ and with low disease activity (LDA) defined as DAS28-CRP $<3.2$, were described over time. For patients entering withdrawal, time to first RA flare during withdrawal and predictors of time to flare were analysed using a Cox proportional-hazards model including the following parameters at 
initial study entry: randomised treatment, DAS28-CRP, swollen joint count, Patient Global Assessment of Disease Activity (PtGA), corticosteroid use, RA symptom duration, smoking status and anti-CCP2 antibody status.

Summary statistics were generated for the mean change from re-treatment baseline to the end of re-treatment in DAS28-CRP and Health Assessment Questionnaire-Disability Index (HAQ-DI) scores, and for proportions of patients with DAS28-CRP remission and LDA at the end of re-treatment. Re-treatment baseline was the last assessment within 30 days before the first re-treatment dose. DAS28-CRP was also summarised by original treatment group, where baseline was the day of starting re-treatment. The 95\% CIs for mean change was based on a t-test. Achievement of DAS28-CRP remission at the end of re-treatment was tested using an adjusted logistic regression model, including the same parameters used for the Cox proportional-hazards model.

Deaths, serious adverse events (SAEs) and serious infections were summarised over the withdrawal and re-treatment periods. Overall infection rates were compared over all study periods.

\section{RESULTS}

Patient disposition and baseline characteristics

Of 351 randomised patients, 290 (82.6\%) completed the treatment period and $225(64.1 \%)$ entered the withdrawal period after achieving LDA, with 176/225 (78.2\%) being in remission. Of the 225 patients, 172 (76.4\%) discontinued the withdrawal phase due to RA flare, of whom 146/172 (84.9\%) entered and 140/146 (95.9\%) completed the re-treatment period. Six months after the initiation of the withdrawal period (month 18), 17/115 $(14.8 \%), 14 / 113(12.4 \%)$ and $9 / 115(7.8 \%)$ patients in the abatacept plus methotrexate, abatacept and methotrexate arms, respectively, had a sustained remission.

For patients who entered the withdrawal and re-treatment periods, baseline characteristics (table 1) were similar to those of the original randomised population. ${ }^{6}$

\section{Efficacy: withdrawal period}

Proportions of patients maintaining DAS28-CRP-defined drug-free remission remained numerically higher in the original abatacept plus methotrexate and abatacept arms versus methotrexate arm to day 253 of withdrawal (month 21: 15/73 (20.5\%), 11/50 (22.0\%) and 11/53 $(20.8 \%)$, respectively). At the end of the withdrawal period (month 24), the number of patients still in remission was very low and similar across the treatment arms: 9/73 (12.3\%) with abatacept plus methotrexate, $7 / 50$ $(14.0 \%)$ with abatacept and 6/53 (11.3\%) with methotrexate (online supplementary figure $\mathrm{S} 1$ ). The same results were seen when using alternative definitions of disease remission: CDAI, SDAI and Boolean remission, and LDA (online supplementary table S1).

Patients who experienced a flare during the withdrawal period and subsequently entered the re-treatment period
( $\mathrm{n}=146)$ were studied to determine if any characteristic at study entry could predict an earlier flare after treatment withdrawal. Kaplan-Meier curves for time to protocol-defined first flare during withdrawal showed no clear differences between randomisation arms (figure 2). Cox proportional-hazards model of time to first flare after treatment withdrawal using various baseline parameters identified that only corticosteroid use at baseline was associated with a faster time to flare $(\mathrm{p}=0.0159$; table 2$)$.

\section{Efficacy: re-treatment period}

As noted, only patients who experienced a flare between month 3 and month 12 of the withdrawal period entered the re-treatment period; a high proportion of these patients entered the re-treatment period and had evaluable efficacy data. Overall, 120 and 106 patients had DAS28-CRP and HAQ-DI assessments, respectively, within 30 days before the first re-treatment dose and after 6 months of re-treatment.

During re-treatment, mean (SD) DAS28-CRP decreased from 5.28 (1.37) to $2.41(0.94)$; mean change (95\% CI) -2.87 (-3.13 to -2.60$)$. There were no notable differences in mean improvement in DAS28-CRP by initial treatment arm (online supplementary table S2). After 6 months' re-treatment, most evaluable patients $(76.6 \% ; 95 / 124)$ had reached LDA (DAS28-CRP <3.2), with 62.9\% $(78 / 124)$ achieving remission (DAS28-CRP $<2.6)$. Mean (SD) HAQ-DI scores decreased during re-treatment: 1.40 (0.63) versus $0.64(0.57)$; mean change $(95 \% \mathrm{CI})-0.76$ $(-0.88$ to -0.64$)$.

Using an adjusted logistic regression model with the same baseline characteristics used to identify factors that could predict faster time to flare, only PtGA at study entry was associated with subsequent achievement of DAS28-CRP remission after re-treatment $(\mathrm{p}=0.0199$; table 3).

\section{Safety}

Two deaths were reported during the study, both in the methotrexate arm during withdrawal (due to respiratory failure and uterine neoplasm/renal failure). Neither was considered by the investigators to be related to study treatment.

During withdrawal, 7/225 patients had an SAE; two in the abatacept plus methotrexate arm (prostate cancer and postprocedural complication) and five in the methotrexate arm (foot deformity, sciatica, respiratory failure, uterine neoplasm and renal failure, and pyelonephritis and urinary tract infection (all $n=1)$ ). None were considered to be related to study treatment. During re-treatment, 4/146 patients had an SAE (tendon rupture, cholangitis, anaemia and abatacept overdose). Only abatacept overdose was considered related to the study drug.

Infection rates were low during the withdrawal and re-treatment periods, particularly when compared with the initial treatment period (table 4). No marked differences were seen between original treatment groups in 


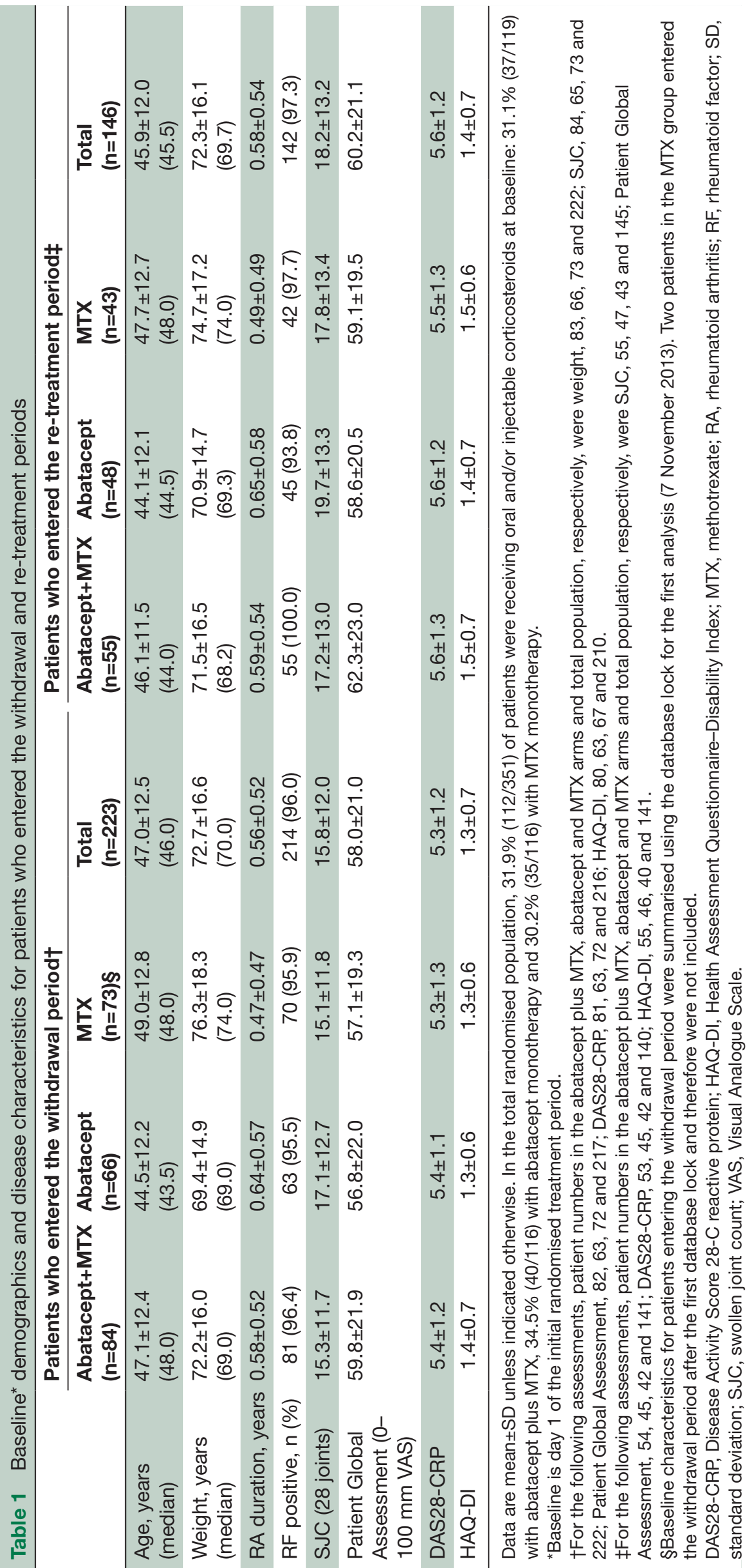




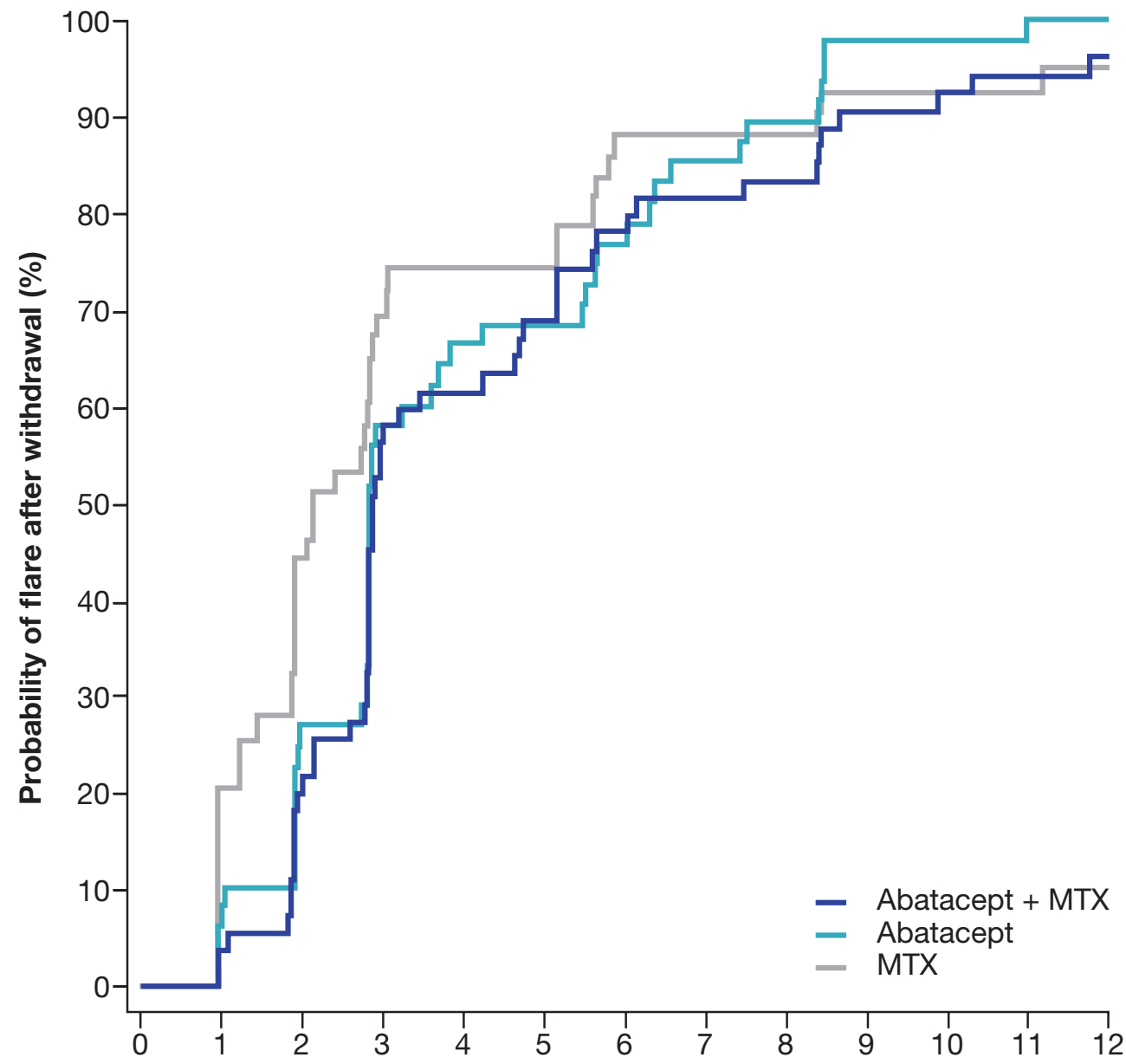

Months in withdrawal period

Patients $(\mathrm{n})$ at risk

$\begin{array}{llllllllllllll}\text { Abatacept + MTX } & 55 & 53 & 44 & 24 & 21 & 17 & 12 & 10 & 9 & 5 & 4 & 3 & 2 \\ \text { Abatacept } & 48 & 45 & 35 & 23 & 16 & 15 & 11 & 7 & 5 & 1 & 1 & 0 & 0 \\ \text { MTX } & 43 & 34 & 24 & 13 & 11 & 11 & 5 & 5 & 5 & 3 & 3 & 3 & 2\end{array}$

Figure 2 Cox proportional-hazards model of time to first RA flare after treatment withdrawal for patients who entered the re-treatment period $(n=146)$. Patients who experienced a flare during the withdrawal period and subsequently entered the retreatment period were included in this analysis. Kaplan-Meier curves for time to protocol-defined first flare during withdrawal are shown. The Cox proportional-hazards model included the following baseline parameters: randomised treatment, DAS28CRP, swollen joint count, Patient Global Assessment of Disease Activity, corticosteroid use, RA symptom duration, smoking status and anticyclic citrullinated peptide 2 antibody status. RA flare was defined as $\geq 2$ of doubling of tender and swollen joint counts from month 12, increase in DAS28-CRP $\geq 1.2$ from month 12 or investigator's clinical judgement of RA. DAS28-CRP, Disease Activity Score $28-C$ reactive protein; MTX, methotrexate; RA, rheumatoid arthritis.

the rate or type of infections. One serious infection was reported during treatment withdrawal (pyelonephritis in the methotrexate arm; 337.4 patient-years) and none during re-treatment (292.2 patient-years).

\section{DISCUSSION}

AVERT investigated the feasibility of drug withdrawal in early RA, and employed the rapid withdrawal of all RA therapies and a strict definition of disease flare. This approach is not recommended in clinical practice. To our knowledge, this is the only controlled study in which the efficacy and safety of re-treatment have been examined.
This unique analysis showed that re-treatment with abatacept plus methotrexate in patients experiencing disease flare after treatment withdrawal could restore remission or LDA in many patients.

During the 12 months following treatment withdrawal, most patients could not remain in drug-free remission, with many experiencing a flare within 6 months. Benefits of abatacept plus methotrexate versus methotrexate alone at 6 months after treatment withdrawal ${ }^{6}$ did not persist over the second 6 months off treatment. Population selection criteria for severe disease used in this study-early, active disease and poor prognostic 
Table 2 Cox proportional-hazards model of time to RA flare after study medication withdrawal ${ }^{\star}$

\begin{tabular}{|c|c|c|}
\hline Baseline parameter & HR (95\% CI) & $P$ value \\
\hline \multicolumn{3}{|l|}{ Treatment } \\
\hline Abatacept+MTX vs MTX & 0.71 (0.46 to 1.09$)$ & 0.1171 \\
\hline Abatacept vs MTX & 0.80 (0.51 to 1.25$)$ & 0.3276 \\
\hline DAS28-CRP & 0.84 (0.64 to 1.11$)$ & 0.2231 \\
\hline SJC (28 joints) & $1.02(1.00$ to 1.04$)$ & 0.1060 \\
\hline PtGA (0-100 mm VAS) & 1.00 (0.99 to 1.01$)$ & 0.9692 \\
\hline $\begin{array}{l}\text { Baseline corticosteroid } \\
\text { use (yes vs no) }\end{array}$ & 1.71 (1.11 to 2.63 ) & 0.0159 \\
\hline $\begin{array}{l}\text { RA symptom duration ( }<3 \\
\text { vs } \geq 3 \text { months) }\end{array}$ & 0.97 (0.66 to 1.42$)$ & 0.8588 \\
\hline $\begin{array}{l}\text { Smoking status (yes } † \text { vs } \\
\text { no) }\end{array}$ & 0.91 (0.63 to 1.33$)$ & 0.6376 \\
\hline $\begin{array}{l}\text { Anti-CCP2 (positive vs } \\
\text { negative) }\end{array}$ & 1.06 (0.73 to 1.54$)$ & 0.7543 \\
\hline
\end{tabular}

HRs and $p$ values were based on a Cox proportional-hazards model including the following baseline parameters: randomised treatment, DAS28-CRP, SJC, PtGA, corticosteroid use, RA symptom duration, smoking status and anti-CCP2 antibody status. RA flare was defined as $\geq 2$ of doubling of tender and swollen joint counts from month 12 , increase in DAS28-CRP $\geq 1.2$ from month 12 or investigator's clinical judgement of RA.

*All randomised and treated patients who entered the withdrawal period $(n=146)$.

†Includes previous and current smokers.

Anti-CCP2, anticyclic citrullinated peptide 2; DAS28-CRP, Disease Activity Score 28-C reactive protein ; MTX, methotrexate; PtGA, Patient Global Assessment of Disease Activity; RA, rheumatoid arthritis; SJC, swollen joint count; VAS, Visual Analogue Scale.

indicators (anticitrullinated peptide antibody (ACPA) positive $)^{6}$ - were inadequate to discern long-term drugfree remission. Only corticosteroid use at study entry was associated with shorter time to flare after treatment withdrawal, which may reflect greater disease severity in patients using corticosteroids or the difficulties associated with corticosteroid withdrawal. ${ }^{6}$ Notably, in ADJUST, a phase II double-blind treatment withdrawal trial, patients with undifferentiated or very early RA and ACPA positivity were treated with intravenous abatacept or placebo for 6 months, followed by treatment discontinuation. Patients receiving abatacept showed delayed disease progression and prolonged inhibition of radiographic progression after cessation of treatment versus placebo, and a decrease from baseline in rheumatoid factor and ACPA titres (however, the titres failed to reach the limits of normal) ${ }^{9}$

Most patients experiencing a flare entered the re-treatment period, during which treatment with abatacept plus methotrexate notably reduced disease activity and improved physical function. LDA was recaptured in three quarters of patients, with two-thirds regaining remission. This is notable given the relatively high disease activity, as captured by the mean DAS28-CRP values, of study patients at the time of flare. Only PtGA predicted
Table 3 Predictive factors for the achievement of DAS28CRP remission at the end of the re-treatment period ${ }^{*}$

\begin{tabular}{|c|c|c|}
\hline Baseline parameter & OR $(95 \% \mathrm{Cl})$ & $P$ value \\
\hline \multicolumn{3}{|l|}{ Treatment } \\
\hline $\begin{array}{l}\text { Abatacept }++ \text { MTX vs } \\
\text { MTX }\end{array}$ & 1.71 (0.63 to 4.63$)$ & 0.6923 \\
\hline Abatacept vs MTX & 2.07 (0.74 to 5.77$)$ & 0.3026 \\
\hline DAS28-CRP & 0.80 (0.44 to 1.47$)$ & 0.4727 \\
\hline SJC (28 joints) & 1.00 (0.96 to 1.05$)$ & 0.9271 \\
\hline PtGA (0-100 mm VAS) & $0.97(0.94$ to 1.00$) \dagger$ & 0.0199 \\
\hline $\begin{array}{l}\text { Baseline corticosteroid use } \\
\text { (yes vs no) }\end{array}$ & 1.34 (0.55 to 3.28) & 0.5219 \\
\hline $\begin{array}{l}\text { RA symptom duration ( }<3 \\
\text { vs } \geq 3 \text { months) }\end{array}$ & $1.08(0.44$ to 2.66$)$ & 0.8634 \\
\hline $\begin{array}{l}\text { Smoking status (yes } \ddagger \text { vs } \\
\text { no) }\end{array}$ & 1.16 (0.49 to 2.79$)$ & 0.7346 \\
\hline $\begin{array}{l}\text { Anti-CCP2 (positive vs } \\
\text { negative)§ }\end{array}$ & $1.62(0.69$ to 3.80$)$ & 0.2730 \\
\hline
\end{tabular}

ORs and $p$ values were based on an adjusted logistic regression model including the following baseline parameters: randomised treatment, DAS28-CRP, SJC, PtGA, corticosteroid use, RA symptom duration, smoking status and anti-CCP2 antibody status.

*All randomised and treated patients who entered the withdrawal period.

†Due to rounding, the upper $95 \% \mathrm{Cl}$ value of 0.995 is shown as 1.00 .

†Includes previous and current smokers.

$\S$ At the end of the treatment period, respectively, 8/98 (8\%), $1 / 85(1 \%)$ and $1 / 94(1 \%)$ of patients treated with abatacept+methotrexate, abatacept and methotrexate became anti-CCP2 seronegative.

Anti-CCP2, anticyclic citrullinated peptide 2; DAS28-CRP, Disease Activity Score 28-C reactive protein; MTX, methotrexate; PtGA, Patient Global Assessment of Disease Activity; RA, rheumatoid arthritis; SJC, swollen joint count; VAS, Visual Analogue Scale.

recapture of remission during re-treatment, suggesting that patients are the best judges of their own disease.

Very few SAEs were reported in the withdrawal or re-treatment periods. For the first time to our knowledge, lower infection rates were demonstrated in the withdrawal and re-treatment periods versus the initial treatment period. Infection rates in RA studies are usually regarded as an indicator of disease-modifying antirheumatic drug (DMARD)-associated toxicity. However, infection rates, including serious infections, probably reflect a complex interplay between the RA state, the disease-activity level and all therapies employed. ${ }^{10}{ }^{11}$ The observed infection rate in the withdrawal period (table 3) may simply reflect the absence of DMARD use. However, this does not explain the relatively similar rate of infection during re-treatment, notably in the group initially receiving methotrexate, without previous exposure to abatacept. A linear relationship was demonstrated between serious infection rates and disease activity, with infection rates and disease activity tending to decrease over time on treatment. ${ }^{12}$ Further understanding of the 
Table 4 Exposure-adjusted incidence rates of infection per 100 patient-years during the treatment, withdrawal and retreatment periods

\begin{tabular}{lcccc}
\hline & $\begin{array}{l}\text { Treatment months } \\
(\mathbf{n = 3 5 1 )}\end{array}$ & $\begin{array}{l}\text { Treatment months } \\
\mathbf{6 - 1 2} \\
(\mathbf{n = 3 5 1 )}\end{array}$ & $\begin{array}{l}\text { Withdrawal } \\
(\mathbf{n = 2 2 5})\end{array}$ & $\begin{array}{l}\text { Re-treatment } \\
(\mathbf{n}=\mathbf{1 4 6})\end{array}$ \\
\hline Overall rate & - & - & $8.9^{*}$ & $16.1 \dagger$ \\
\hline Abatacept+MTX & 116.6 & 64.6 & 7.2 & 17.2 \\
Abatacept & 126.1 & 66.5 & 6.0 & 9.3 \\
MTX & 110.7 & 72.8 & 13.5 & 22.3 \\
\hline
\end{tabular}

*All patients who entered the withdrawal period.

†All patients who experienced protocol-defined rheumatoid arthritis flare after treatment withdrawal, entered the re-treatment period and received open-label abatacept+MTX.

MTX, methotrexate.

factors influencing the development of infections and their interactions will assist in designing better, less toxic treatment strategies. The relative safety of withdrawal and re-treatment in this setting can, however, be stressed.

Limitations of this analysis include the high discontinuation rate before the second 6 months of the withdrawal period. At study conception, a formal definition of flare was unavailable; therefore, the designers formulated their own robust definition. However, the Outcome Measures in Rheumatology Clinical Trials RA Flare Core Domain Set is now available ${ }^{13}$ and may have use in future clinical trials and practice by testing domains using the RA-flare questionnaire. ${ }^{14}$

This analysis demonstrates that, after receiving only 12 months of DMARD therapy before withdrawal of all RA treatments, few patients with severe early RA can maintain drug-free remission over 12 months. However, for those experiencing disease flare after treatment withdrawal, this unique investigation showed that re-treatment with abatacept plus methotrexate can restore remission or LDA in many patients, with good tolerability.

\section{Author affiliations}

'Leeds Institute of Rheumatic and Musculoskeletal Medicine, University of Leeds, Leeds, UK

${ }^{2}$ NIHR Leeds Musculoskeletal Biomedical Research Centre, Leeds Teaching Hospitals NHS Trust, Leeds, UK

${ }^{3}$ Department of Rheumatology and Clinical Immunology, Charité - University Medicine Berlin, Berlin, Germany

${ }^{4}$ Division of Rheumatology, Hospital for Special Surgery, New York, New York, USA ${ }^{5}$ Department of Rheumatology, CHU Montpellier, Montpellier University, Montpellier, France

${ }^{6}$ Division of Rheumatology, University of California Los Angeles, Los Angeles, California, USA

${ }^{7}$ Immunoscience, Bristol-Myers Squibb, Princeton, New Jersey, USA

${ }^{8}$ Department of Rheumatology, Leiden University Medical Center, Leiden, Netherlands

Acknowledgements The authors thank Sandra Overfield (Bristol-Myers Squibb) for co-ordination of the study. Professional medical writing and editorial assistance was provided by Sharon Gladwin, PhD, at Caudex and was funded by Bristol-Myers Squibb.

Contributors All authors were involved in the conception and design of the study, data acquisition and/or interpretation, drafting and critical revision of the manuscript, and final approval of the version to be published, and agree to be accountable for all aspects of the work.

Funding This study was funded by Bristol-Myers Squibb.
Competing interests PE: grant/research support: AbbVie, Merck, Pfizer, Roche; consultant: AbbVie, Bristol-Myers Squibb, Merck, Pfizer, Roche, Lilly, Novartis, Samsung Bioepis. GRB: grant/research support: clinical trials for Bristol-Myers Squibb, AbbVie, Pfizer, Medimmune, Novartis, Roche, UCB, Lilly; consultant: BristolMyers Squibb, AbbVie, Pfizer, MSD, Medlmmune, Roche, UCB; speakers bureau: Bristol-Myers Squibb, AbbVie, Pfizer, MSD, Roche, UCB. VPB: grant/research support: Amgen, Pfizer, Bristol-Myers Squibb, Janssen, UCB, Sanofi, Roche/ Genentech; consultant: Amgen, AbbVie, Pfizer, Bristol-Myers Squibb, UCB, Roche, Regeneron. BGC: grant/research support: Pfizer, Roche-Chugai, UCB; honoraria/ speakers bureau: AbbVie, Bristol-Myers Squibb, Merck, Pfizer, Roche-Chugai, UCB, Janssen, Lilly, Novartis, Sanofi. DEF: grant/research support: Amgen, Bristol-Myers Squibb, Pfizer, Roche/Genentech; consultant: AbbVie, Amgen, Bristol-Myers Squibb, Corbus, Cytori, Novartis, Pfizer, Roche/Genentech; speakers bureau: AbbVie, BristolMyers Squibb. MAM: shareholder and employee: Bristol-Myers Squibb. TWJH: grant/research support: EU and Dutch Arthritis Foundation; consultant: Abbott Laboratories, Biotest AG, Bristol-Myers Squibb, Crescendo Bioscience, Novartis, Pfizer, Roche, Sanofi, Schering-Plough, UCB, Eli Lilly; speakers bureau: Abbott Laboratories, Biotest AG, Bristol-Myers Squibb, Novartis, Pfizer, Roche, Sanofi, Schering-Plough.

Patient consent for publication All patients or their legally acceptable representatives gave written, informed consent prior to study entry.

Ethics approval The AVERT study protocol was approved by the Institutional Review Board or Independent Ethics Committee at each site.

Provenance and peer review Not commissioned; externally peer reviewed.

Data sharing statement BMS policy on data sharing may be found online (https:// www.bms.com/researchers-and-partners/clinical-trials-and-research/disclosurecommitment.html).

Open access This is an open access article distributed in accordance with the Creative Commons Attribution Non Commercial (CC BY-NC 4.0) license, which permits others to distribute, remix, adapt, build upon this work non-commercially, and license their derivative works on different terms, provided the original work is properly cited, appropriate credit is given, any changes made indicated, and the use is non-commercial. See: http://creativecommons.org/licenses/by-nc/4.0/.

\section{REFERENCES}

1. Cush JJ. Early rheumatoid arthritis - is there a window of opportunity? J Rheumatol Suppl 2007;80:1-7.

2. van Nies JA, Krabben A, Schoones JW, et al. What is the evidence for the presence of a therapeutic window of opportunity in rheumatoid arthritis? A systematic literature review. Ann Rheum Dis 2014;73:861-70.

3. Nagy G, van Vollenhoven RF. Sustained biologic-free and drug-free remission in rheumatoid arthritis, where are we now? Arthritis Res Ther 2015;17:181.

4. Nam JL. Rheumatoid arthritis management of early disease. Curr Opin Rheumatol 2016;28:267-74.

5. Nam JL, Takase-Minegishi K, Ramiro S, et al. Efficacy of biological disease-modifying antirheumatic drugs: a systematic literature review informing the 2016 update of the EULAR recommendations for the management of rheumatoid arthritis. Ann Rheum Dis 2017;76:1113-36.

6. Emery P, Burmester GR, Bykerk VP, et al. Evaluating drug-free remission with abatacept in early rheumatoid arthritis: results from 
the phase $3 \mathrm{~b}$, multicentre, randomised, active-controlled AVERT study of 24 months, with a 12-month, double-blind treatment period. Ann Rheum Dis 2015;74:19-26.

7. Peterfy C, Burmester GR, Bykerk VP, et al. Sustained improvements in MRI outcomes with abatacept following the withdrawal of all treatments in patients with early, progressive rheumatoid arthritis. Ann Rheum Dis 2016;75:1501-5.

8. Felson DT, Smolen JS, Wells G, et al. American College of Rheumatology/European League Against Rheumatism provisional definition of remission in rheumatoid arthritis for clinical trials. Ann Rheum Dis 2011;70:404-13.

9. Emery P, Durez P, Dougados M, et al. Impact of T-cell costimulation modulation in patients with undifferentiated inflammatory arthritis or very early rheumatoid arthritis: a clinical and imaging study of abatacept (the adjust trial). Ann Rheum Dis 2010;69:510-6.

10. Singh JA. Infections with biologics in rheumatoid arthritis and related conditions: a scoping review of serious or hospitalized infections in observational studies. Curr Rheumatol Rep 2016;18:61.
11. Dixon WG, Watson $\mathrm{K}$, Lunt $\mathrm{M}$, et al. Rates of serious infection, including site-specific and bacterial intracellular infection, in rheumatoid arthritis patients receiving anti-tumor necrosis factor therapy: results from the British Society for Rheumatology Biologics Register. Arthritis Rheum 2006;54:2368-76.

12. Emery P, Gallo G, Boyd H, et al. Association between disease activity and risk of serious infections in subjects with rheumatoid arthritis treated with etanercept or disease-modifying anti-rheumatic drugs. Clin Exp Rheumatol 2014;32:653-60.

13. Bykerk VP, Bingham CO, Choy EH, et al. Identifying flares in rheumatoid arthritis: reliability and construct validation of the OMERACT RA flare core domain set. RMD Open 2016;2:e000225.

14. Bartlett SJ, Hewlett S, Bingham CO, et al. Identifying core domains to assess flare in rheumatoid arthritis: an OMERACT international patient and provider combined Delphi consensus. Ann Rheum Dis 2012;71:1855-60. 